\title{
Cumulative Gradient Based Image Sharpness Evaluation Algorithm For Auto Focus Control of Thermal Imagers
}

\author{
Anurag Kumar Srivastava ${ }^{\star}$, and Neeta Kandpal ${ }^{* *}$ \\ *Instruments Research \& Development Establishment, Dehradun-248008, India, anuragvns@gmail.com \\ **Instruments Research \& Development Establishment, Dehradun-248008, India, neeta@irde.drdo.in
}

\begin{abstract}
An auto-focus system brings a de-focused image into focus by automatically adjusting the focus lens assembly to capture the sharpest image based on sharpness evaluation. It is built around an image sharpness evaluation module and a control module. Image sharpness evaluation module computes the degree of focus in an image and provides the required data to the control module for controlling the focus lens assembly to achieve the auto focus functionality. An Image Sharpness Evaluation algorithm based on cumulative gradient for auto-focus control of thermal imagers is presented in this paper which involves passive auto-focusing approach.
\end{abstract}

Keywords: Thermal Imager, Auto Focus, Cumulative Gradient, MATLAB, Sharpness, ACM, Noise

\section{Introduction}

An auto-focus system brings a de-focused image into focus by automatically adjusting the focus lens assembly to capture the sharpest image based on sharpness evaluation. Depending on the scene distance from the thermal imager, the focusing lens is required to be adjusted for obtaining sharp focused image [1, 8]. Focus can be achieved manually, or automatically using an active or passive approach. Active methods are based on emitting a sound wave or an infrared signal in order to estimate the distance of focusing lens assembly from target, and then by using the measured distance the appropriate lens position is calculated. Passive methods rely solely on the captured image evaluation methods.

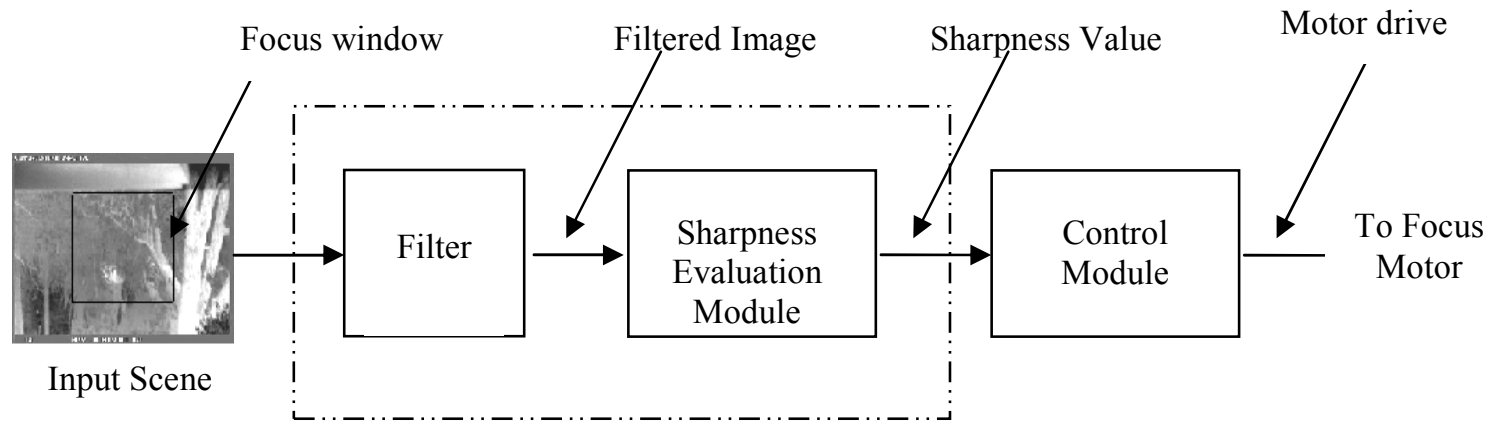

Fig1. Auto focus approach block diagram

A passive auto-focusing system consists of three modules (fig. 1). A filtering module is used to increase the SNR of the input scene. Median filtering [7] has been employed since it offers the best combination between performance, effective noise reduction, and preservation of object features in a scene. To generate the filtered image, $(3 \times 3)$ size sliding neighborhood window approach has been followed to replace the center pixel of the window by median of each pixel of the sliding window. After filtering, the sharpness evaluation module computes the "sharpness value" of the filtered image. This value indicates the degree of focus of the input scene. Sharpness values are computed for images acquired at different focus lens assembly positions. It is computed for a central window of image to decrease the time required for capturing and processing an image. These sharpness values are used by the control module to control the focusing lens assembly to make it reach at the position where the image sharpness is maximum [6]. The focus lens assembly is powered electrically to reach the position where the computed image sharpness value is maximum. 
Plot of sharpness values corresponding to different focus lens assembly positions gives the sharpness function. A good sharpness function should work for all possible scene conditions and it should be monotonic in nature with respect to the image focusing state [7]. The more defocused the image is, the less its value should be. Moreover, a good sharpness function should have good discrimination power; i.e. it should give a sharper response when the focus point is closer and it should be able to combat noisy and low-contrast imaging conditions [7].

\section{Sharpness Evaluation Algorithm}

Sharpness of an image is linked to the sharpness of its edges. Focused images contain more sharp edges than defocused images. This approach involves computing a sharpness value that reflects the sharp edges within a selected region of image. Gradient measure has been employed which senses the transition rate between adjacent pixel values. Sharper edges in focused images lead to a sharper transition in pixel values resulting in larger gradient value for that image as compared to the defocused images.

For computing cumulative gradient, a region of image (focus window) is selected. Pixel values corresponding to each column within the selected region of image are summed together and then the sum value is divided by the number of pixels in a column. Averaged Sum of each column is then subtracted from the averaged sum of neighbouring column. For a $[\mathrm{mxn}]$ selected region of image (fig. 2(a)) (where $\mathrm{m}$ is number of rows \& $n$ is number of columns), all ' $m$ ' pixel values within each of the ' $n$ ' columns are summed together to get ' $n$ ' number of sum values. Then the difference between averaged sum value corresponding to a column and the averaged sum value of adjacent column is computed which gives $(n-1)$ difference values. All these difference values are compared and the maximum difference value is taken as the sharpness value for the image frame (fig. 2(b)). This sharpness value increases as the image becomes more focused. Adding column data together and averaging it results in noise reduction as well. This algorithm takes care of vertical edges within the scene as they are more prominent than horizontal edges.

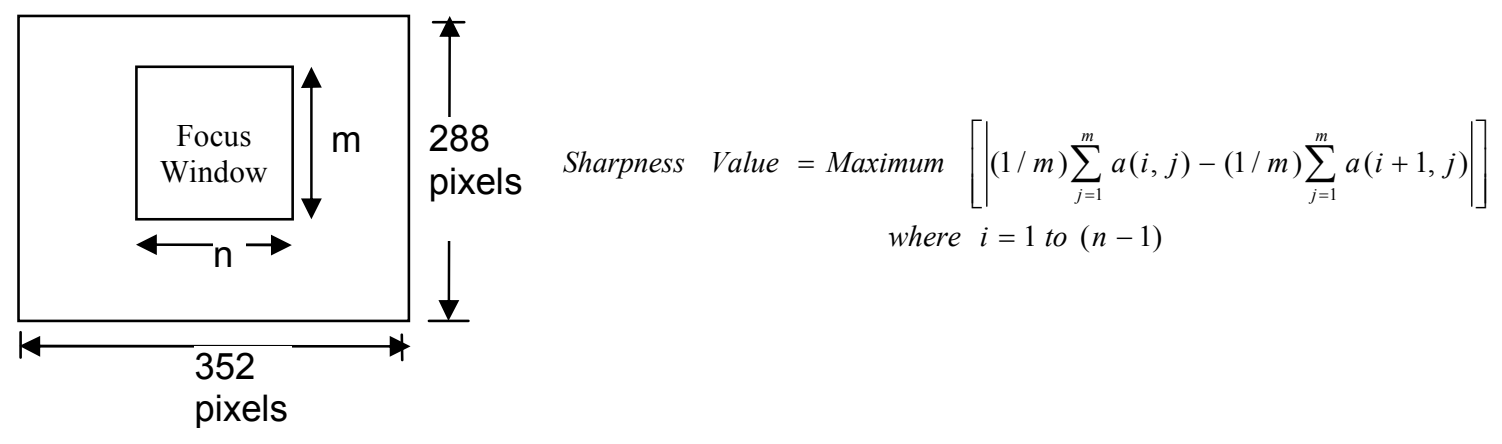

(a)

(b)

Fig2. (a) Central Focus window selection (b) Sharpness evaluation formula

\section{Simulation \& Results}

Video sequences were captured with a thermal Imager mounted on a tripod. These sequences were converted into 'avi' format and frames were extracted from '.avi' file in MATLAB. This particular camera has motorized FOV \& focus control. Video was captured using an mpeg-encoder. The focusing lens assembly was moved from one extreme position to the other extreme position so that the video shows a transition from defocused state to focused state and then again to defocused state. Frames extracted were 8 -bit gray scale images of $288 \times 352$ pixels resolution. Cumulative gradient were computed for central $160 \times 160$ windows of the image frames. In actual auto focus scenario the extracted image frames refer to images at different focus lens assembly positions. Sharpness values corresponding to different frames were plotted to give the sharpness function. Other algorithms viz. Standard Deviation [2, 3], Absolute Central Moment [2, 3] were applied on same images and sharpness functions for these algorithms were compared with the sharpness function of cumulative gradient algorithm. 8 frames (fig. 3(a)) were extracted from video sequence 1 and sharpness values were computed for $160 \times 160$ central portions of all the frames. 


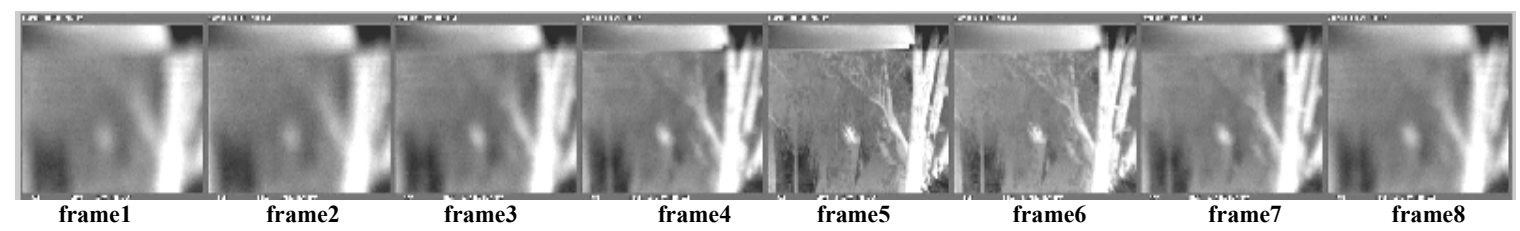

(a)

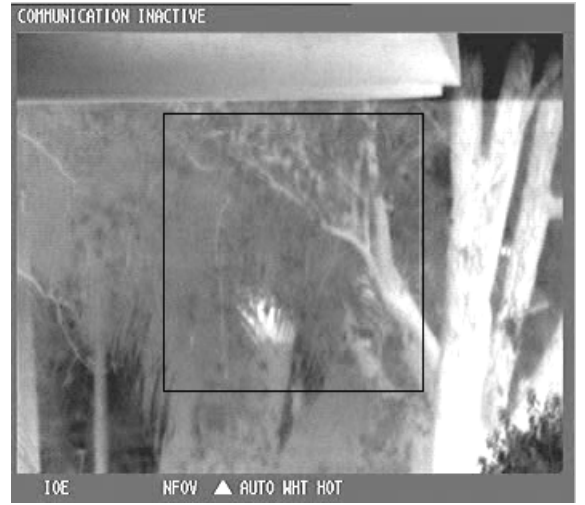

(b)

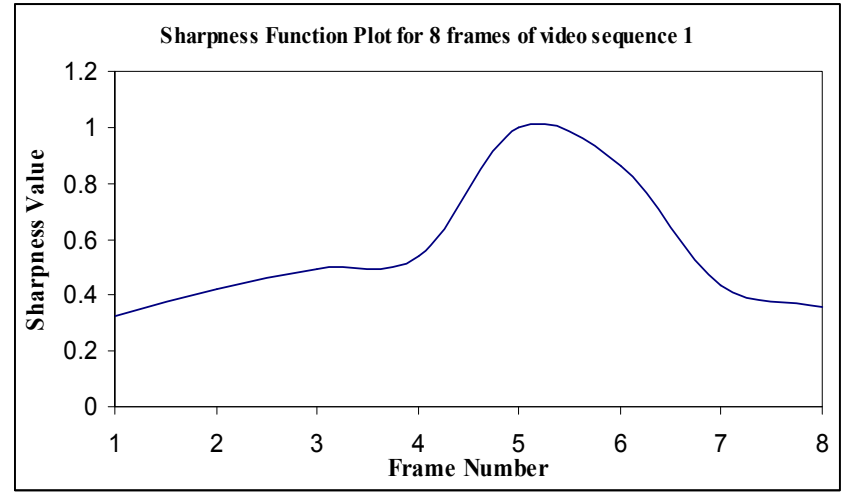

(c)

Fig3 (a) 8 frames of video sequence 1 in different focusing states (b) Best focused frame (frame 5) with focus window area highlighted (c) Sharpness function plot for 8 sequential frames of video sequence 1

The sharpness value was found maximum for frame no. 5 (fig. 3(b)). Sharpness function plot in fig. 3 (c) shows large variation range of 0.3 to 1.0 which is very important for the control module to work efficiently.

Same algorithm was applied to 8 frames of two other video sequences. The simulation results are shown below:

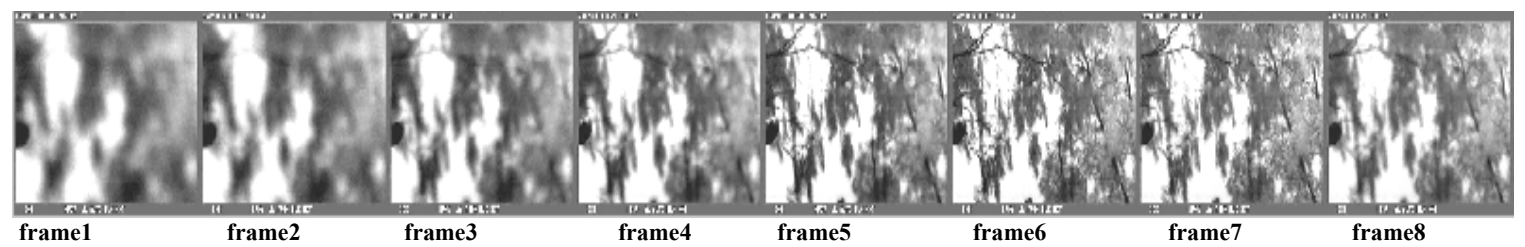

(a)

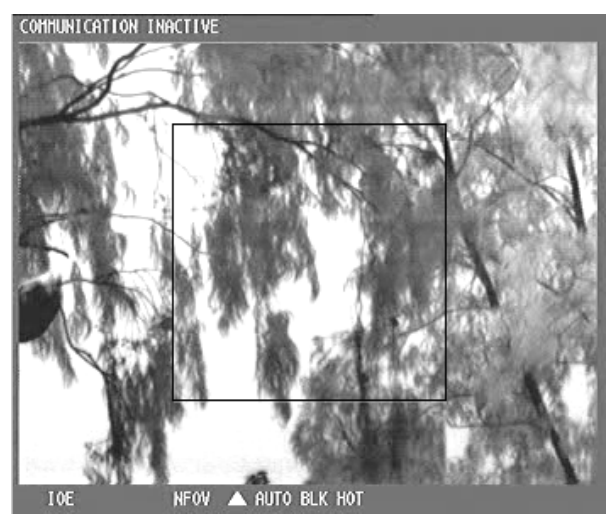

(b)

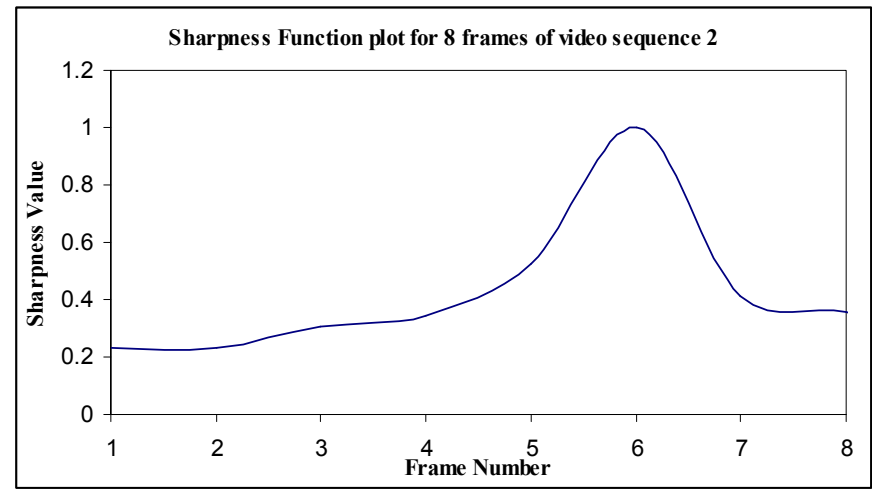

(c)

Fig4 (a) 8 frames of video sequence 2 in different focusing states (b) Best focused image: frame no. 6 (c) Sharpness function plot for 8 sequential frames of video sequence 2 


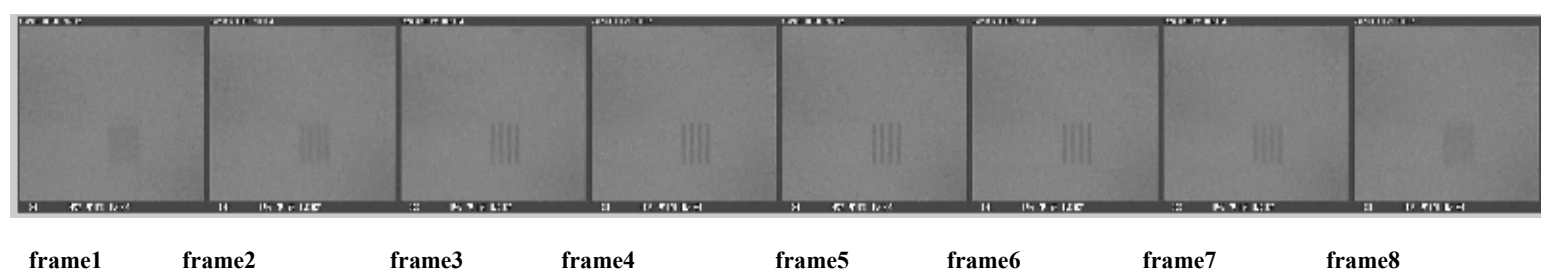

(a)

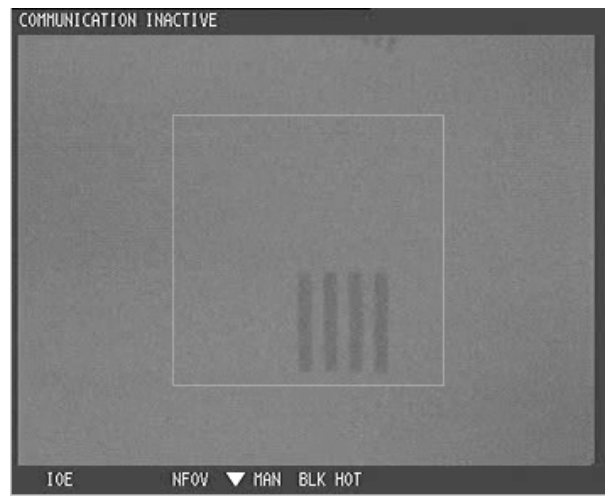

(b)

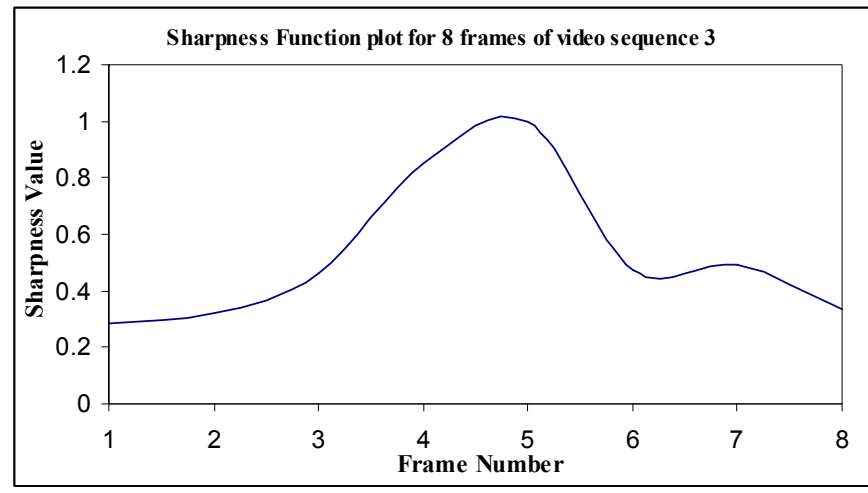

(c)

Fig5 (a) 8 frames of video sequence 3 in different focusing states (b) Best focused image: frame no. 5

(c) Sharpness function plot for 8 sequential frames of video sequence 3

The simulation results show that the sharpness functions, for all 3 video sequences, have one peak at the best focus position and it strictly decreases away from this peak.

The sharpness values were calculated for 3 different center focus windows of sizes $80 \times 80$, $160 \times 160$ and $240 \times 240$ of video sequence 1 (fig. 6(a)). Larger focus window size resulted in smoother sharpness function (fig. 6(b)). The best focused frame was found the same (frame 5) for all three focus windows selected.

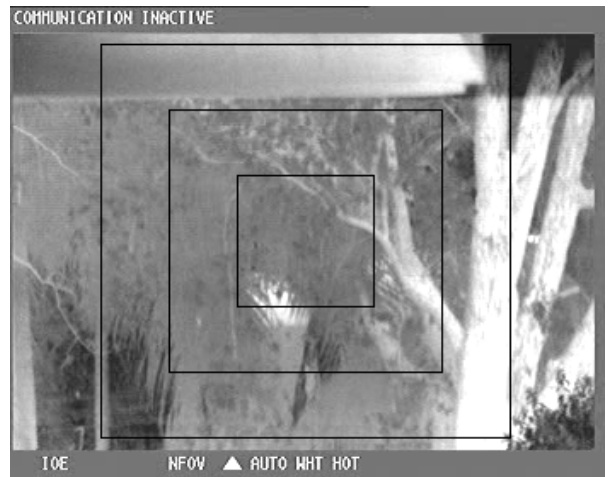

(a)

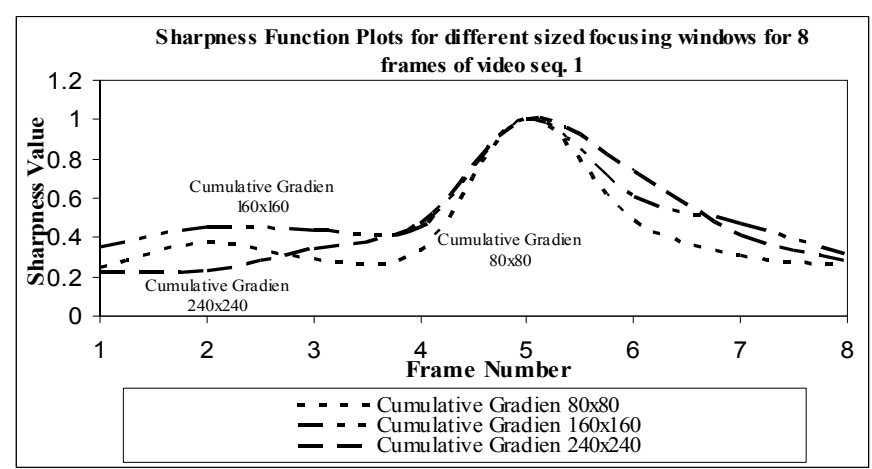

(b)

Fig6 (a) Focused Frame of video seq. 1 with 3 different sized focus windows. (b) Sharpness function plots for different focus window sizes for 8 frames of video sequence 1

To see the effect of noise on the algorithm results, noise was added to images extracted from video sequence 1 using MATLAB "imnoise (im,'speckle',0.05)" function. Sharpness values were calculated for noise added video sequence 1 for the two center focus windows of sizes $160 \times 160$ and 240x240. The images were filtered by a median filter and sharpness values were computed for both focus windows sizes. 


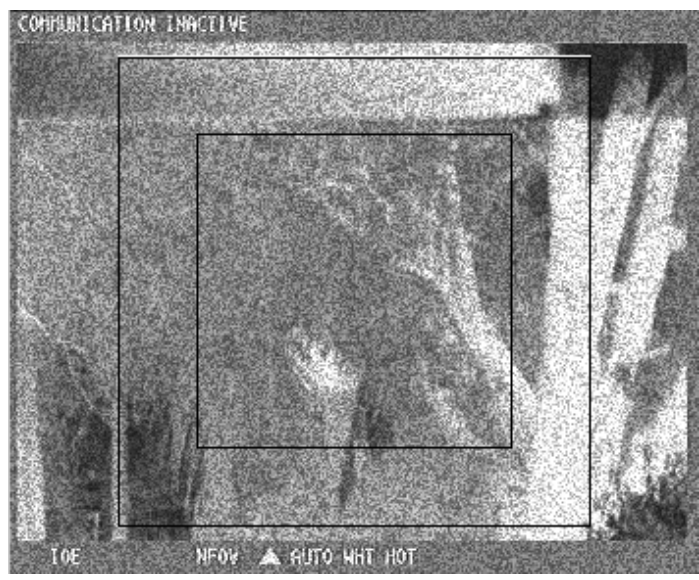

(a)

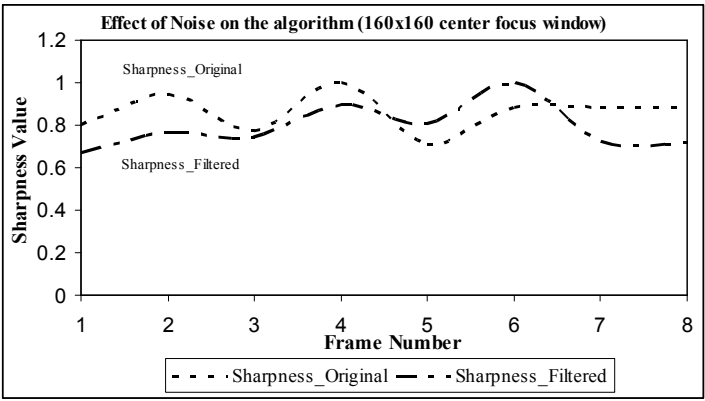

(c)

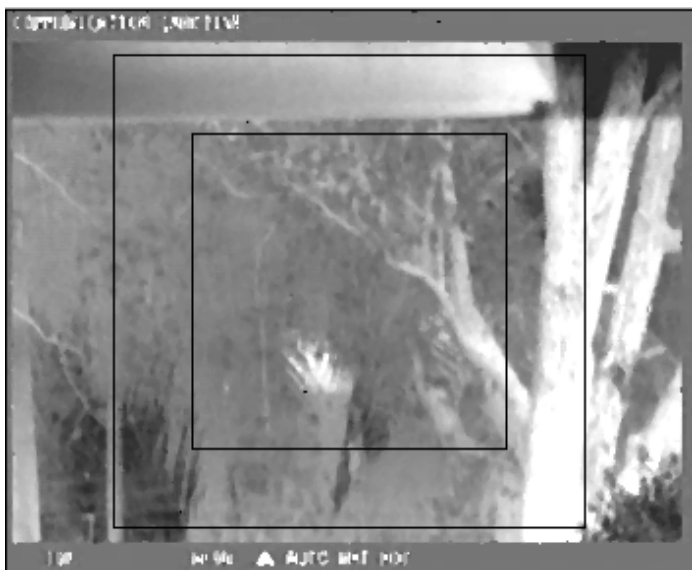

(b)

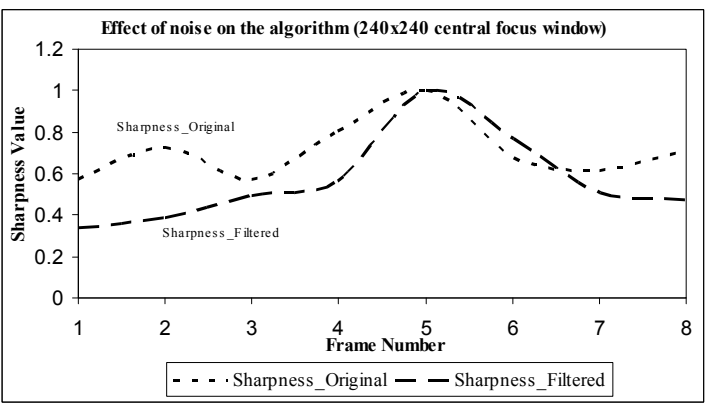

(d)

Fig7 (a) Noise added best focused frame of video seq. 1 with two focus windows highlighted (b) Filtered frame with two focus windows highlighted (c) Sharpness function plots for $160 \times 160$ center focus window size of noise added \& filtered 8 frames of video sequence 1 (d) Sharpness function plots for $240 \times 240$ center focus window size of noise added \& filtered 8 frames of video sequence 1

The sharpness function for $240 \times 240$ center focus window was found to have larger variation range of sharpness values (fig. 7(d)) as compared to the $160 \times 160$ focus window size (fig.7(c)). Filtering resulted in a smoother sharpness curve. $240 \times 240$ center focus window size was found effective in combating this much amount of noise and identifying the best focused frame correctly. With the advances in image acquisition \& processing, full frame size can also be computed for the sharpness value which will result in more noise robustness.

The cumulative gradient algorithm was compared with two other sharpness evaluation algorithms of standard deviation (STD_DEV) \& absolute central moment (ACM). Sharpness values corresponding to all 3 algorithms were calculated for central $160 \times 160$ portions of 8 sequential frames (fig. 8(a)) \& 8 filtered frames (fig. $8(b))$ of video sequence 1 . All three sharpness functions were scaled to $(0,1)$ range so that the results can be compared. Cumulative gradient algorithm was found to be having greater variation range of sharpness values as compared to the variation range for other 2 algorithms (fig 8 ). 


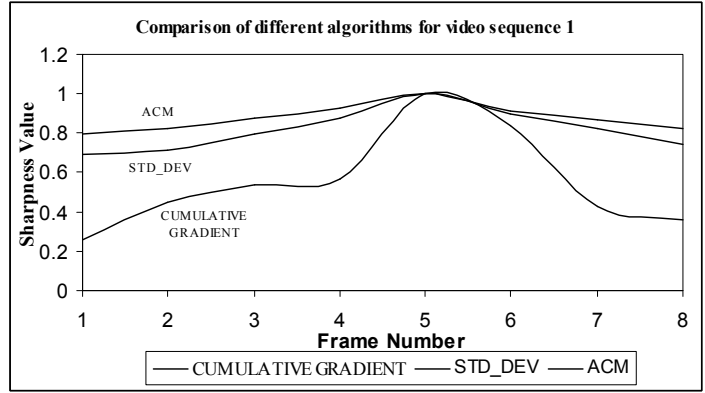

(a)

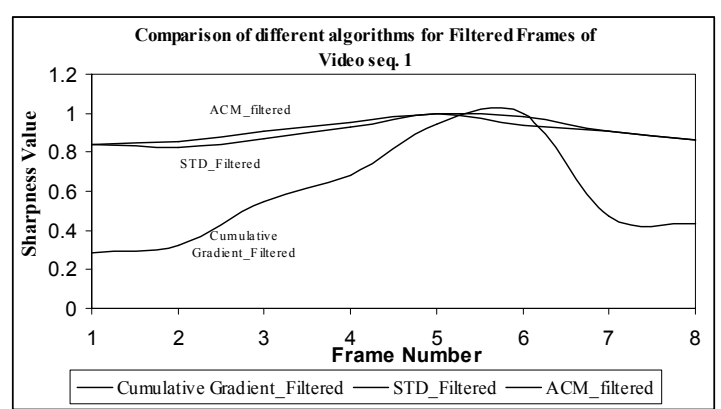

(b)

Fig8. (a) Comparison of 3 algorithms for 8 frames of video sequence 1 (b) Comparison of 3 algorithms for 8 filtered frames of video sequence 1

An accurate and reliable auto focusing system is very important for obtaining good quality sharp images by thermal imagers. Image sharpness evaluation module is the basis to realize an accurate, fast and reliable auto focus system. An image sharpness evaluation method based on the cumulative gradient technique is presented in this paper. This algorithm gives good results with scenes with different sized targets having varying contrast and noise levels. This module along with a control module completes the design of an auto-focus system for thermal imagers. The control module searched for the best focused position using the sharpness values. Being a less computationally intensive algorithm it is easier to implement this image sharpness module in hardware.

\section{Acknowledgement}

We would like to thank Dr. S S Negi, Director, I.R.D.E for the kind permission to publish this paper. We would also like to thank Sh. A K Sahay and Dr. Ajay Kumar, Associate Director, I.R.D.E for the continuous guidance and support during this work.

\section{REFERENCES}

[1] R.D.Hudson, Infrared Systems Engineering, John Wiley, NewYork, 1969.

[2] Feng Li, Hong Jin, "A Fast Auto Focusing Method For Digital Still Camera ",Proceedings of the Fourth International Conference on Machine Learning and Cybernetics, Guangzhou, 18-21 August 2005

[3] Mukul V. Shirvaikar, "An Optimal Measure for Camera Focus and Exposure" Proceedings of the IEEE SSST 2004

[4] Ng Kuang Chern,Nathaniel Poo Aun Neow and Marcelo H. Ang Jr., " Practical issues in pixel-based autofocusing for machine vision", Proceedings of the 2001 IEEE International Conference on Robotics \& Automation, Seoul, Korea, May 21-26, 2001

[5] Chun-Hung Shen and Homer H. Chen, "Robust Focus Measure for Low-Contrast Images", Proceedings of the IEEE SSST 2006

[6] J. Baina and J. Dublet, "Automatic focus and iris control for video cameras", Fifth International Conf. on Image Processing and its Applications, pp. 232-235, July 1995.

[7] Mark Antunes and Michael Trachtenberg, "All-In-Focus Imaging From A Series Of Images On Different Focal Planes" , B.Sc thesis report, Faculty of Engineering, University of Manitoba, March 2005

[8] G.C Holst, "Testing and Evaluation for Infrared Imaging systems", JCD Publishing, Chap. 5 \& 7.

[9] Gregory A. Baxes, "Digital Image Processing- Principles and Applications" , 1994

[10] MATLAB version 12.0 